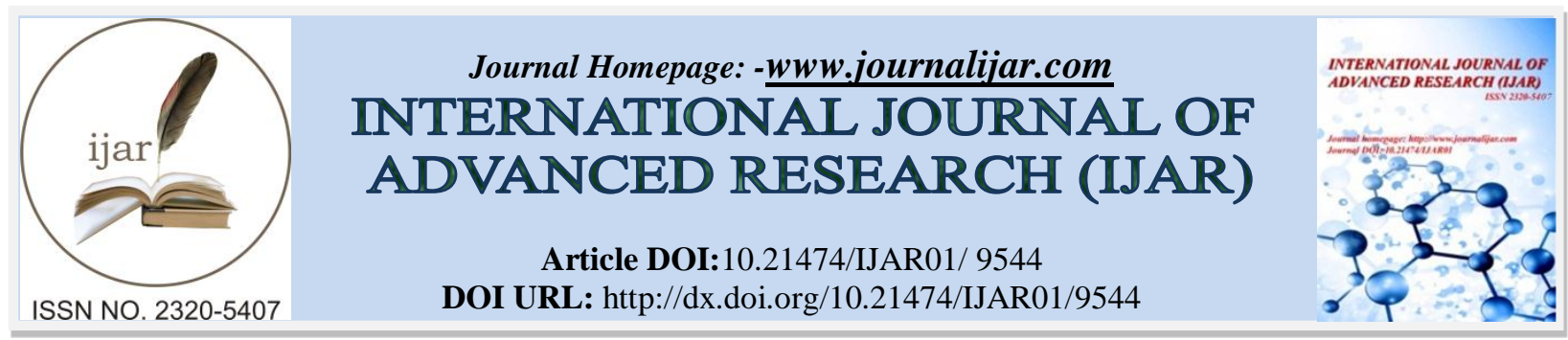

RESEARCH ARTICLE

\title{
A CLINICAL STUDY ON SANDHIGATA VATA W.S.R. TO OSTEOARTHRITIS AND ITS MANAGEMENT WITH AJMODADI VATAK.
}

\section{Fating Vandana ${ }^{1}$,Acharya Ramakant ${ }^{2}$ And Deshmukh Sanjay ${ }^{3}$.}

1. Ph.D Scholor, Samhita Siddhant \& Sanskrit (Basic Principles), Gopbandhu Ayurveda Mahavidyalaya Puri, Odisha.

2. Head of Dept. Samhita Siddhant \& Sanskrit (Basic Principles), Gopbandhu Ayurveda Mahavidyalaya Puri, Odisha.

3. Head of Dept., Rognidan \& Vikriti Vigyan, Rajiv Lochan Ayurved Medical College, Durg (C.G.).

\section{Manuscript Info}

Manuscript History

Received: 08 June 2019

Final Accepted: 10 July 2019

Published: August 2019

Key words:-

Sandhigata Vata, Osteoarthritis, Ajmodadi Vatak.

\section{Abstract}

Ayurveda, the holistic science an upveda of Atharva Veda was incepted by Brahma. The aim of Ayurveda is to maintain the state of good health of healthy person and render cure to the suffering. A healthy life and higher longevity are always desirable. During different stages of human life disease prevalence is common due to genetic, infection, infestations, systemic, environmental, age related issues and so on. Health and disease are two opposite diversions of life. The human body is so peculiar, it decays in every moment. As per ayurveda the life span (ayu) is devided into three parts viz. Balyavastha, , Maddhyavastha, Vriddhavastha ${ }^{1}$. In Vriddhavastha due to Physiological and Pathological changes gradual deterioration, decreasing ability and inactivity occurs. Ayurveda states that in Vriddhavastha all dhatus undergo kshaya leading to Vataprakopa and making individual prone to some diseases. Among them Sandhigata vata is very common. According to world health organization (WHO) Sandhigata vata (Osteoarthritis) is the second commonest musculoskeletal problem in the world population (30\%) after back pain $(50 \%)$. It is occurring in about $20 \%$ the population as a whole and in $50 \%$ of those above the age of 60 yrs. Disease starts between the age of $45-50$ yrs. It is the common cause of disability in an elderly population throughout the world. Considering this and keeping in view the prevalence of this disease. I have been employed through this Clinical Study. I have done Clinical trial work in the management of Sandhigata vata with Ajmodadi Vatak (Herbal formulation) and compared with control drug Piroxicam. In this study total 80 patients were selected, 50 patients were treated with trial drug Ajmodadi Vatak and 30 patients were treated with control drug Piroxicam. Study reveals Ajmodadi Vatak is effective in Sandhigata Vata.

Copy Right, IJAR, 2019,. All rights reserved.
Corresponding Author:- Fating Vandana.

Address:-Ph.D Scholor, Samhita Siddhant \& Sanskrit (Basic Principles), Gopbandhu Ayurveda Mahavidyalaya Puri, Odisha. 


\title{
Introduction:-
}

Sandhigata vata is the most common disease of old age mentioned under vataroga in ayurveda. Symptomatically Sandhigata vata is having close resemblance to osteoarthritis. It occurs due to degeneration of cartilage $\&$ synovial membrane in joints, which affects mostly weight bearing joints i.e. knees \& hips. Common sign \& symptoms are inflammation \& stiffness in joints, pain during movements of joints. Modern science explains it as degenerative joint disease. Bone \& Joints disorders are curse of the health for human population. Though most of them do not cut the years of life but the life of years. Tridosha are the functional integrities of body and their equilibrium keeps the person free from ailments and viceversa. Also it is said that disease are caused due to food for the maintenance of equilibrium within tridosha conductive food is advocated in Ayurveda. If a person is subjected to vatavardhaka ahara it may lead to excitation of vata predominance with the properties of laghu, ruksha, sheeta etc ${ }^{2}$. In Sandhigata vata two things happens- Aggravation of vata and dhatukshaya (asthikshaya). As a consequence loss of sheshmaka cough occurs. The asthi cartilage undergoes degenerative change putting lipping margins leading to formation of osteophytes. Khavaigunya sets in within strotas and in joint cavity through a gradual process. As a result vayu is filled in the empty space, vayu being ruksha dries up shleshmaka kapha and other kaphaj elements like snayu, kandara making the joint dry and causes sandhigata vata. In the allopathic system of medicine the management mainly based on symptomatically relief in painful conditions but still there is need for such therapeutic regimen that could retard the disease progression. For the search of such agents herbal remedies and dietary supplements have become an important area of research. In this present study and herbal formulation i.e. Ajmodadi vatak mentioned in chakradatta has been clinically employed in the treatment of Sandhigata vata ${ }^{3}$.

\section{Aims and objective:-}

1. To evaluate efficacy of Ajmodadi Vatak (Trial drug)

2. To evaluate efficacy of Piroxicam (Control drug)

3. To compare the efficacy of both drugs (Trial \& Control)

\section{Material \& Methods:-}

The study has been carried out in department of Kayachikitsa GAM Puri Odisha, total number of 80 cases of either sex registered for the study were selected from OPD and IPD. Out of 80 cases, 50 patients were exposed to trial drug Ajmodadi vatak and 30 were put under control drug piroxicam.

\section{Inclusion Criteria}

1. A selection criteria was followed on the basis of the classical signs and symptoms and radiological findings

2. Pain in joints, Swelling, Tenderness, Restricted movement, Stiffness of joints, Crepitation, Muscular Wasting

3. Patients between age grp of $40-70$ years.

4. Patients without any anatomical deformity of knee.

\section{Exclusion criteria}

1. Patients below 40 and above 70 years of age.

2. Patients suffering from other diseases like DM, Carcinoma, Tuberculosis, Cardiac disease, Vatarakta etc.

3. Bed ridden patients.

4. Patients using active allopathic medicines for pain $\&$ inflammation.

\author{
Grouping \\ Group A \\ 1. Trial drug - Ajmodadi vatak (Chakradatta Amvatarogadhikar) \\ 2. Dose - $10 \mathrm{~g} /$ day in divided dose \\ 3. Anupan - Luke warm water \\ 4. Duration - 35 days \\ 5. Group B \\ 6. b) Control drug - Piroxicam \\ 7. Dose - 20mg OD (after meal) \\ 8. Duration -7 days
}




\section{Observation}

Clinical trials were conducted on patients who had been thoroughly scrutinized through clinical signs \& symptoms \& X-ray and diagnosed as Sandhigata vata. Among the trial grp patients, the assessment was carried after each 7 days interval i.e. (AT1) on 7th day, (AT2) on 14th day, (AT3) on 21st day, (AT4) on 28th day, (AT5) on 35th day, Control drug was given for 7 days. Before starting the treatment investigations like Hb, TLC, DLC, ESR, RA, serum uric acid were done. After completion of Clinical trial investigations are repeated but no changes were found in their measure inspite of having clinical improvement in signs \& symptoms of osteoarthritis. Blood count \& ESR are normal in osteoarthritis ${ }^{4}$. In the present study knee $\mathrm{x}$-ray was done before and after treatment but no changes was observed in any X-ray. In this clinical study, maximum $48.75 \%$ patients were in 51-60 years of age group, $52.50 \%$ were females, $99 \%$ were married, labour class patients were $38.75 \%, 27.50 \%$ were housewives, vatakapha predominance was found in $57 \%$ Patients, $31 \%$ patients had madhyam koshtha. Course of disease was progressive in $80 \%$ patients, $56 \%$ of patients were having family history of osteoarthritis, $35 \%$ were found in chronic stage , $38.75 \%$ was in menopausal state of life $47.50 \%$ patients were obese. Knee is one of the big weight bearing joints and thus $97.50 \%$ was found knee joint involvement and other joint involved was $2.50 \%$, Consumption of ruksha, sheeta, alpa ahara was found in $52.50 \%$ patients. Excessive physical exercise was found in $47.50 \%$ and excessive fasting was found in $20 \%$ Patients.

\begin{tabular}{|l|l|l|l|l|}
\hline Symptoms wise distribution of 80 Patients of Sandhigata Vata & \multicolumn{2}{l|}{ Total } & \multirow{2}{*}{$\%$} \\
\cline { 1 - 5 } Symptoms & Number of Patients & Control Group B & & \\
\cline { 2 - 5 } & Trial Group A & 30 & 80 & $100.00 \%$ \\
\hline Pain & 50 & 30 & 78 & $97.50 \%$ \\
\hline Tenderness & 48 & 30 & 80 & $100.00 \%$ \\
\hline Swelling & 50 & 27 & 76 & $95.00 \%$ \\
\hline Stiffness & 49 & 18 & 61 & $76.25 \%$ \\
\hline Crepitations & 43 & 10 & 49 & $61.25 \%$ \\
\hline Restricted Movements & 39 & 03 & 08 & $10.00 \%$ \\
\hline Muscular Wasting & 05 & 11 & 39 & $48.75 \%$ \\
\hline Radiological Assessment & 28 & & & \\
\hline
\end{tabular}

Statistical Analysis showing the effectiveness of Trial and control drug to sign and symptoms

\begin{tabular}{|c|c|c|c|c|c|c|c|c|}
\hline $\begin{array}{l}\text { Sign } \\
\text { Symptoms }\end{array}$ & $\begin{array}{l}\text { \&Treatment } \\
\text { Group }\end{array}$ & $\begin{array}{l}\text { BT \& } \\
\text { AT }\end{array}$ & Mean \pm SD & SEM & Df & $\mathrm{t}$-value & $\mathrm{p}$-value & Remarks \\
\hline \multirow{8}{*}{ Pain } & \multirow{6}{*}{$\begin{array}{l}\text { Ajmodadi } \\
\text { VaTak }\end{array}$} & BT & $1.32 \pm .551$ & .078 & \multirow{6}{*}{50} & & & \\
\hline & & AT1 & $1.28 \pm .454$ & .064 & & 1.429 & $>0.05$ & Non Significant \\
\hline & & AT2 & $1.10 \pm .678$ & .096 & & 3.718 & $<0.01$ & Highly Significant \\
\hline & & AT3 & $.90 \pm .789$ & .112 & & 5.957 & $<0.0001$ & $\begin{array}{l}\text { Extremely } \\
\text { Significant }\end{array}$ \\
\hline & & AT4 & $.54 \pm .676$ & .096 & & 11.870 & $<0.0001$ & $\begin{array}{l}\text { Extremely } \\
\text { Significant }\end{array}$ \\
\hline & & AT5 & $.28 \pm .640$ & .091 & & 13.797 & $<0.0001$ & $\begin{array}{l}\text { Extremely } \\
\text { Significant }\end{array}$ \\
\hline & \multirow[t]{2}{*}{ Piroxicam } & BT & $1.17 \pm .461$ & .084 & \multirow[t]{2}{*}{30} & & & \\
\hline & & AT & $.03 \pm .183$ & .033 & & 17.954 & $<0.0001$ & $\begin{array}{l}\text { Extremely } \\
\text { Significant }\end{array}$ \\
\hline \multirow{8}{*}{ Tenderness } & \multirow{6}{*}{$\begin{array}{l}\text { Ajmodadi } \\
\text { VaTak }\end{array}$} & BT & $1.52 \pm .614$ & .087 & \multirow{6}{*}{50} & & & \\
\hline & & AT1 & $1.44 \pm .705$ & .100 & & 2.064 & $<0.05$ & Significant \\
\hline & & AT2 & $1.38 \pm .667$ & .094 & & 2.824 & $<0.01$ & Highly Significant \\
\hline & & AT3 & $1.28 \pm .701$ & .099 & & 3.934 & $<0.0001$ & $\begin{array}{l}\text { Extremely } \\
\text { Significant }\end{array}$ \\
\hline & & AT4 & $1.10 \pm .707$ & . 100 & & 5.521 & $<0.0001$ & $\begin{array}{l}\text { Extremely } \\
\text { Significant }\end{array}$ \\
\hline & & AT5 & $1.32 \pm .551$ & .078 & & 2.646 & $<0.05$ & Significant \\
\hline & \multirow[t]{2}{*}{ Piroxicam } & BT & $1.23 \pm .430$ & .079 & \multirow[t]{2}{*}{30} & & & \\
\hline & & AT & $.17 \pm .379$ & .069 & & 23.028 & $<0.0001$ & Extremely \\
\hline
\end{tabular}




\begin{tabular}{|c|c|c|c|c|c|c|c|c|}
\hline & & & & & & & & Significant \\
\hline \multirow{8}{*}{ Swelling } & \multirow{6}{*}{$\begin{array}{l}\text { Ajmodadi } \\
\text { VaTak }\end{array}$} & BT & $1.48 \pm .677$ & .096 & \multirow{6}{*}{48} & & & \\
\hline & & AT1 & $1.46 \pm .676$ & .096 & & 1.000 & $>0.05$ & Non Significant \\
\hline & & AT2 & $1.32 \pm .683$ & .097 & & 3.055 & $<0.01$ & Highly Significant \\
\hline & & AT3 & $1.04 \pm .638$ & .090 & & 5.755 & $<0.0001$ & $\begin{array}{l}\text { Extremely } \\
\text { Significant }\end{array}$ \\
\hline & & AT4 & $.82 \pm .629$ & .089 & & 8.985 & $<0.0001$ & $\begin{array}{l}\text { Extremely } \\
\text { Significant }\end{array}$ \\
\hline & & AT5 & $.60 \pm .639$ & .090 & & 9.028 & $<0.0001$ & $\begin{array}{l}\text { Extremely } \\
\text { Significant }\end{array}$ \\
\hline & Piroxicam & BT & $1.20 \pm .484$ & .088 & 30 & & & \\
\hline & & AT & $.07 \pm .254$ & .046 & & 17.954 & $<0.0001$ & $\begin{array}{l}\text { Extremely } \\
\text { Significant }\end{array}$ \\
\hline \multirow{8}{*}{ Stiffness } & \multirow{6}{*}{$\begin{array}{l}\text { Ajmodadi } \\
\text { VaTak }\end{array}$} & BT & $1.58 \pm .538$ & .076 & \multirow{6}{*}{49} & & & \\
\hline & & AT1 & $1.52 \pm .544$ & .077 & & 1.769 & $>0.05$ & Non Significant \\
\hline & & AT2 & $1.48 \pm .544$ & .077 & & 2.333 & $<0.05$ & Significant \\
\hline & & AT3 & $1.38 \pm .667$ & .094 & & 3.500 & $<0.01$ & Highly Significant \\
\hline & & AT4 & $1.32 \pm .713$ & .101 & & 3.775 & $<0.001$ & $\begin{array}{l}\text { Extremely } \\
\text { Significant }\end{array}$ \\
\hline & & AT5 & $1.20 \pm .756$ & .107 & & 5.067 & $<0.0001$ & $\begin{array}{l}\text { Extremely } \\
\text { Significant }\end{array}$ \\
\hline & \multirow[t]{2}{*}{ Piroxicam } & BT & $1.13 \pm .571$ & .104 & \multirow[t]{2}{*}{27} & & & \\
\hline & & AT & $.07 \pm .254$ & .046 & & 11.217 & $<0.0001$ & $\begin{array}{l}\text { Extremely } \\
\text { Significant }\end{array}$ \\
\hline \multirow{8}{*}{ Crepitation } & \multirow{6}{*}{$\begin{array}{l}\text { Ajmodadi } \\
\text { VaTak }\end{array}$} & BT & $1.06 \pm 0.586$ & 0.083 & \multirow{6}{*}{43} & & & \\
\hline & & AT1 & $1.04 \pm 0.605$ & 0.086 & & 1.00 & $>0.05$ & Non Significant \\
\hline & & AT2 & $1.04 \pm 0.605$ & 0.086 & & 1.00 & $>0.05$ & Non Significant \\
\hline & & AT3 & $1.02 \pm 0.622$ & 0.088 & & 1.429 & $>0.05$ & Non Significant \\
\hline & & AT4 & $1.00 \pm 0.639$ & 0.090 & & 1.769 & $>0.05$ & Non Significant \\
\hline & & AT5 & $1.00 \pm 0.639$ & 0.090 & & 1.769 & $>0.05$ & Non Significant \\
\hline & \multirow[t]{2}{*}{ Piroxicam } & BT & $0.73 \pm 0.691$ & 0.126 & \multirow[t]{2}{*}{18} & & & \\
\hline & & AT & $0.50 \pm 0.572$ & 0.104 & & 2.971 & $<0.01$ & Highly Significant \\
\hline \multirow{8}{*}{$\begin{array}{l}\text { Restricted } \\
\text { Movement }\end{array}$} & \multirow{6}{*}{$\begin{array}{l}\text { Ajmodadi } \\
\text { VaTak }\end{array}$} & BT & $0.88 \pm 0.558$ & 0.079 & \multirow{6}{*}{39} & & & \\
\hline & & AT1 & $0.80 \pm 0.571$ & 0.079 & & 2.064 & $<0.05$ & Significant \\
\hline & & AT2 & $0.80 \pm 0.571$ & 0.079 & & 2.061 & $>0.05$ & Non Significant \\
\hline & & AT3 & $0.76 \pm 0.591$ & 0.084 & & 2.585 & $<0.05$ & Non Significant \\
\hline & & AT4 & $0.76 \pm 0.591$ & 0.084 & & 2.585 & $<0.05$ & Non Significant \\
\hline & & AT5 & 0.000 & 0.000 & & 11.143 & $<0.0001$ & $\begin{array}{l}\text { Extremely } \\
\text { Significant }\end{array}$ \\
\hline & \multirow[t]{2}{*}{ Piroxicam } & BT & $0.40 \pm 0.621$ & 0.113 & \multirow[t]{2}{*}{10} & & & \\
\hline & & AT & $0.17 \pm 0.379$ & 0.069 & & 2.971 & $<0.01$ & Highly Significant \\
\hline \multirow{8}{*}{$\begin{array}{l}\text { Muscular } \\
\text { Wasting }\end{array}$} & \multirow{6}{*}{$\begin{array}{l}\text { Ajmodadi } \\
\text { VaTak }\end{array}$} & BT & $0.14 \pm 0.452$ & 0.064 & \multirow{6}{*}{05} & & & \\
\hline & & AT1 & $0.14 \pm 0.452$ & 0.064 & & 0.000 & $>0.05$ & Non Significant \\
\hline & & AT2 & $0.14 \pm 0.452$ & 0.064 & & 0.000 & $>0.05$ & Non Significant \\
\hline & & AT3 & $0.14 \pm 0.452$ & 0.064 & & 0.000 & $>0.05$ & Non Significant \\
\hline & & AT4 & $0.14 \pm 0.452$ & 0.064 & & 0.000 & $>0.05$ & Non Significant \\
\hline & & AT5 & $0.12 \pm 0.435$ & 0.062 & & 1.000 & $>0.05$ & Non Significant \\
\hline & Piroxicam & BT & $0.13 \pm 0.434$ & 0.079 & 03 & & & \\
\hline & & AT & $0.10 \pm 0.403$ & 0.074 & & 1.000 & $>0.05$ & Non Significant \\
\hline & & BT & $0.60 \pm 0.571$ & 0.081 & & & & \\
\hline & & AT1 & $0.60 \pm 0.571$ & 0.081 & & 0.000 & $>0.05$ & Non Significant \\
\hline & Ajmodadi & AT2 & $0.60 \pm 0.571$ & 0.081 & & 0.000 & $>0.05$ & Non Significant \\
\hline Radiological & VaTak & AT3 & $0.60 \pm 0.571$ & 0.081 & 28 & 0.000 & $>0.05$ & Non Significant \\
\hline & & AT4 & $0.58 \pm 0.575$ & 0.081 & & 0.573 & $>0.05$ & Non Significant \\
\hline
\end{tabular}




\begin{tabular}{|c|c|c|c|c|c|c|c|}
\hline \multirow{3}{*}{ 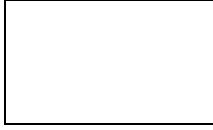 } & AT5 & $0.54 \pm 0.579$ & 0.082 & & 1.353 & $>0.05$ & Non Significant \\
\hline & BT & $0.37 \pm 0.49$ & 0.089 & \multirow[t]{2}{*}{11} & & & \\
\hline & AT & $0.37 \pm 0.49$ & 0.089 & & 0.000 & $>0.05$ & Non Significant \\
\hline
\end{tabular}

\section{Discussion:-}

1. In this study maximum $48.75 \%$ patients were in 51-60 years of age grp. Almost all person by age 40 have some pathologic change in weight bearing joint. Sandhigata Vata is the Vatavyadhi which mainly occurs in vriddhavastha due to aggravation of vata \& dhatukshaya. After age 50 which is declining stage of madhyavaya symptoms like joint pain \& swelling started appearing commonly \& it becomes prominent by age 70 .

2. $52.50 \%$ were female patients, which indicates that Sandhigata vata is more common in female and here the lack of female hormone (oestrogen) in the menopausal period also plays an important role. Modern thought also reflect the same type of prevalence ${ }^{5}$. Also in menopausal state of life due to Dhatukshaya aggravation of vayu occurs that causes Sandhigata vata.

3. 52.50\% patients did not have regular exercise in their routine life. Lack of exercise gradually leads to the weight gain which exerts pressure on weight bearing joints which ultimately leads to Sandhigata vata. Osteoarthritis mainly occurs in the weight bearing joints Data shows 11 pound weight reduction, reduce $50 \%$ risk of osteoarthritis. $5 \%$ weight loss in over weight patients gives $18 \%$ gain in overall function ${ }^{6}$. So weight reduction is very important in case of osteoarthritis.

4. Among the other causative factors in $47.50 \%$, intake of ruksha, sheeta \& alpa ahara were also found. These nidan played an important role in aggravation of vayu and thus causes Sandhigata vata.

\begin{tabular}{|c|c|c|c|c|c|c|c|c|c|c|c|c|c|}
\hline \multicolumn{14}{|c|}{ Total effect of therapy } \\
\hline \multirow{3}{*}{$\begin{array}{l}\text { Sr. } \\
\text { No. }\end{array}$} & \multirow{3}{*}{$\begin{array}{l}\text { Clinical } \\
\text { assessment }\end{array}$} & \multicolumn{10}{|c|}{ Trial Grp } & \multirow{2}{*}{\multicolumn{2}{|c|}{$\begin{array}{l}\text { Control } \\
\text { Grp } \\
\text { AT(7days) }\end{array}$}} \\
\hline & & \multicolumn{2}{|c|}{$\begin{array}{l}\text { AT1 } \\
\text { (7days) }\end{array}$} & \multicolumn{2}{|c|}{$\begin{array}{l}\text { AT2 } \\
\text { (14days) }\end{array}$} & \multicolumn{2}{|c|}{$\begin{array}{l}\text { AT3(21da } \\
\text { ys) }\end{array}$} & \multicolumn{2}{|c|}{ AT4(28days) } & \multicolumn{2}{|c|}{$\begin{array}{l}\text { AT5(35days } \\
\text { ) }\end{array}$} & & \\
\hline & & $\mathbf{F}$ & $\%$ & $\mathbf{F}$ & $\%$ & $\mathbf{F}$ & $\%$ & $\mathbf{F}$ & $\%$ & $\mathbf{F}$ & $\%$ & $\mathbf{F}$ & $\%$ \\
\hline 1 & $\begin{array}{l}\text { Maximum } \\
\text { Improvement } \\
(75-100 \%)\end{array}$ & - & - & - & - & 05 & 10 & 08 & 16 & 13 & 26 & 27 & 90 \\
\hline 2 & $\begin{array}{l}\text { Moderate } \\
\text { Improvement } \\
(>50 \text { to } 75 \%)\end{array}$ & - & - & 01 & 02 & 03 & 06 & 12 & 24 & 21 & 42 & 03 & 10 \\
\hline 3 & $\begin{array}{l}\text { Mild } \\
\text { Improvement } \\
(>25 \text { to } 50 \%)\end{array}$ & 03 & 06 & 14 & 28 & 18 & 36 & 22 & 44 & 12 & 24 & - & - \\
\hline 4 & $\begin{array}{l}\text { No } \\
\text { Improvement } \\
(<25 \%)\end{array}$ & 47 & 94 & 35 & 70 & 24 & 48 & 08 & 16 & 04 & 08 & - & - \\
\hline
\end{tabular}

\section{Probable Mechanism of trial drug}

Probable Mechanism of trial drug Ajmodadi vatak is having 6 ingredient cedrus deodara, piper nigrum, embelia ribes \& anethum sowa which possess anti-inflammatory activity and 4 ingredients piper nigrum, embelia ribes, cedrus deodara \& zingiber officinale are having analgesic properties. (Maximum ingredients are KaphavataShamak) which reduces swelling and pacifies pain. The combination of piper longum, piper nigrum and zingiber officinal, known in ayurveda as trikatu is a significant bio enhancer. Some ingredients like piper longum, Embelia ribes, Plumbego Zeylanica, Argyria speciosa, old jaggery are having rasayana property can delay the further degeneration process in Sandhigata Vata.

Ajmodadi vatak is also having properties like ushna, snigdha, Vatashamak which helps in pacification of aggravated vata and subsides the pain. On the overall effect of Ajmodadi Vatak is anti inflammatory, analgesic and immunomodulatory which reduces inflammation, subside the pain, reduces Stiffness and improves joint mobility and help prevent degenerative changes in Sandhi (Joints) ${ }^{7-14}$. 


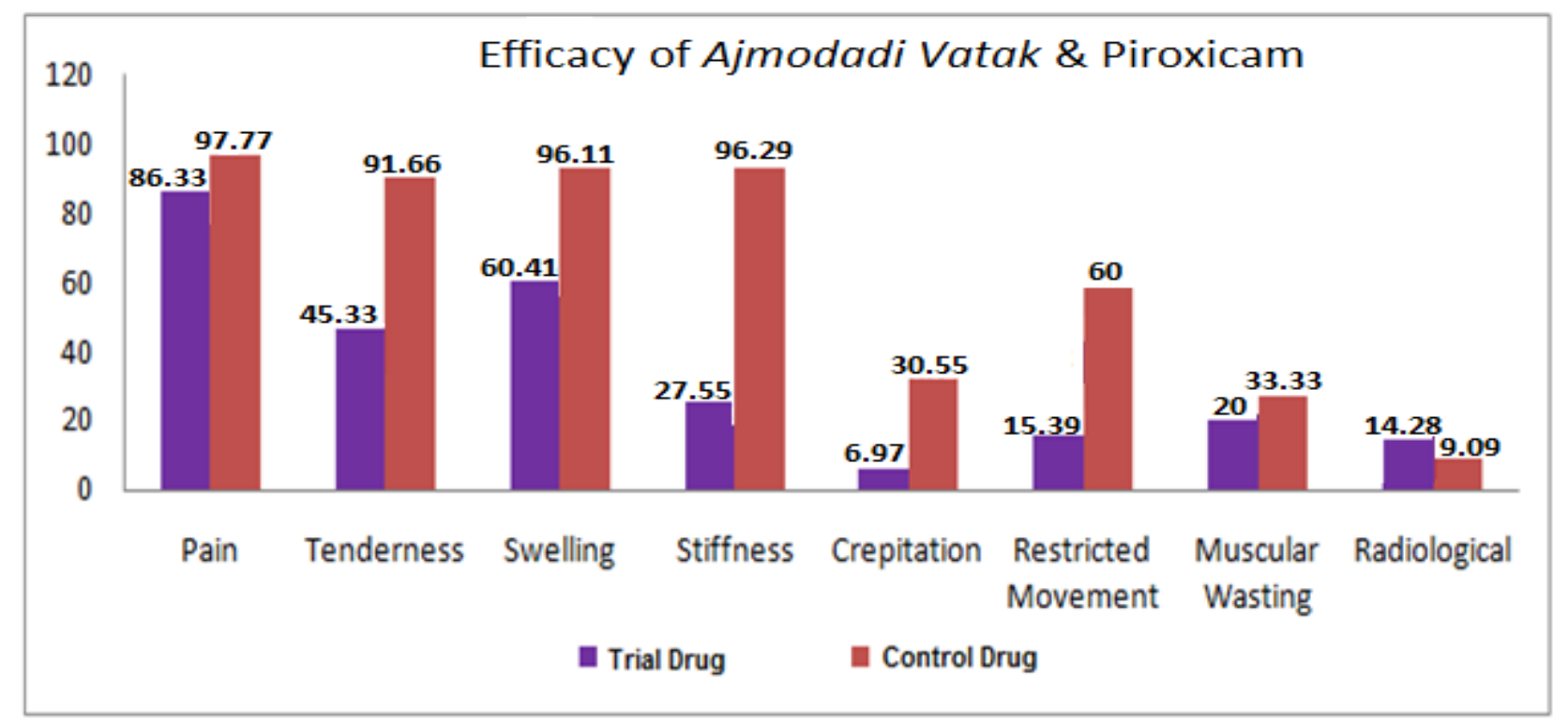

Comparision of percentage efficacy (\%) of trial drug \& control drug Treatment in Sandhigata Vata

\section{Conclusion:-}

Sandhigata Vata is the commonest form of articular disorder which occurs in vriddhavastha which limits everyday activities making patients disabled, Vata dosha plays main role. Allopathic treatment has its own limitation in managing this disease with troublesome side effects. Whereas such type of condition can be better treatable by the management mentioned in Ayurvedic Classics. So this clinical study is carried out with ajmodadi vatak a herbal formulation mentioned in Chakradatta as effective in vata vyadhi. In present study data shows that Ajmodadi Vatak is an effective remedy in Sandhigata Vata (Osteoarthritis) as it has potent analgesic \& anti inflammatory effects also it renders immunomodulatory effects in body which aids in arresting the progress of the disease. In both groups (A \& B), $26 \%$ \& 90\% patients achieved maximum improvement, $42 \%$ \& $10 \%$ patients were having moderate improvement and in grp A 24 patients found in mild improvement. There was no apparent change was observed in $\mathrm{X}$-ray before and after treatment. So similar type of assignments should be continued to make use for longer duration pinpointing on the radiological progression. Present study reveals that the selected management have potential effect on Sandhigata Vata with added advantage of being free from side effects as compared to control drug.

\section{References:-}

1. Kaviraj Ambika dutta Shastri, Sushrut Samhita, Part I Hindi commentry, Chaukhambha Sanskrit Sansthan Varanasi, Edition 2009.

2. Acharya Siddhi Nandan Mishra, Charak Samhita, Chaukhambha Orientalia Varanasi, Hindi Commentary, Chakrapanidatta Chikitsasthan 2009.

3. Panditraj Vaidya Ravidatta Shastri, Chakradatta Probodhini, Hindi Vyakhya, Chaukhambha Surbharti Prakashan 2006, Amvatarogadhikar - PP119

4. Davidson's Principles \& Practice of medicine, 20th edition (2006) - PP 1100

5. Dr. C.C. Chatterjee, Human Physiology, Medical Allied Agency Calcutta, Edition 2002, Vol II, Ch-4, PP 265

6. Harrison's Principles of internal medicine, 15th edition (2003) - PP 1988

7. Shinde U.A., Phadke A.S., Nair A.M., Mungantiwar A.A., Dikshin V.J. \& Saraf M.N. (1999) Studies on the anti inflammatory and analgesic activity of cedrus deodara (Roxb.) Loud, wood oil, J. Ethnopharmacol Vol.65, PP 21-27

8. Chandraseker HI (1982) clinical trial on rheumatoid arthritis with vyoshadi guggulu and vachadi kwatha, rheumatism vol. 17(4), PP 127-130

9. Sharma P.C., Yelne M.B. \& Dennis T.J., Database on medicinal plants used in Ayurveda vol. 5, CCRAR 2002, PP 480

10. Atal C.K., Siddique M.A., Zutshi U, Amla V, Johri R.K., Rao P.G. \& Kour S. (1984) non narcotic, orally effective centrally acting analgesic from an Ayurvedic drug J. Ethnopharma co/ Vol. 11(3) PP 309-317 
11. Vaz, Z.R., Mata L.V. \& Calixto J.K. (1997) Analgesic effect of the herbal medicine catuama in thermal and chemical models of nociception in mice. Phytotherapy Res. Vol. 11(2), pp 101-106

12. Bansal P., Bhardwaj R.K., Tyagi R.K. \& Gupta S.K. comparative antinoception and anti-inflammatory profile of nimesulide vs nimedulide and piperine combination (XXXl Ann. Conf. Indian Pharmacol soc. Lucknow, Dec. 19-20th 1998) Indian J. Pharmacol vol. 31(1) PP 79

13. Sharma P.C. Yelne M.B. \& Dennis T.J., Database on medicinal plants used in Ayurveda vol.2, CCRAS 2005, PP 551

14. Sharma P.C., Yelne M.B. \& Dennis T.J. Database on medicinal plants used in Ayurveda vol. 4, CCRAS 2002 PP 189. 